\title{
Maximising the Diagnostic Utility of the Exercise Treadmill Test
}

\author{
Kofi Tekyi Asamoah* \\ National Cardiothoracic Centre, Korle Bu Teaching Hospital, Ghana
}

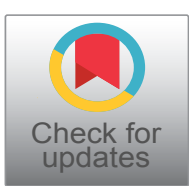

*Corresponding author: Kofi Tekyi Asamoah, National Cardiothoracic Centre, Korle Bu Teaching Hospital, Accra, PO Box KB 846, Ghana, Tel: +233-55-0434-068

\begin{abstract}
Ischaemic heart disease was the leading cause of mortality according to the World Health Organisation in the year 2018 and disease burden keeps increasing steadily across the globe. Early diagnosis and intervention are therefore key in mitigating its effect on society.

The exercise treadmill test is an important, cost-effective investigation employed in the evaluation of coronary artery disease, allowing for early diagnosis and a decision on revascularization to be made. Reliable interpretation depends on multiple factors, combined in the Duke Treadmill Score. An early positive test result suggests a severe coronary artery obstruction which requires urgent revascularisation to reduce morbidity and mortality risk.

This is a report on a patient who had an early positive test, with subsequent angiographic findings validating the exercise treadmill test results. It also highlights relevant considerations in performing and interpreting the exercise treadmill test.
\end{abstract}

\section{Keywords}

Exercise treadmill test, Coronary artery disease, Duke treadmill score, Positive stress test, Ischaemic heart disease

\section{Introduction}

Ischaemic heart disease was reported as the $3^{\text {rd }}$ leading cause of mortality in Singapore in 2014, accounting for approximately $16 \%$ of deaths [1]. The World Health Organisation also named ischaemic heart disease as the leading cause of death worldwide in 2016 [2]. These figures underscore the burden of ischaemic heart disease and highlight the need for prevention, prompt diagnosis and treatment.

Obstructive coronary artery disease (CAD) may be evaluated by a number of tests; the exercise treadmill test (ETT), stress echocardiogram, CT coronary angiogram, myocardial nuclear scanning among others. The ETT has the advantage of estimating the individual's functional capacity, unlike the imaging modalities [3].

The ETT is a useful, non-invasive test that is relatively cost effective $[4,5]$. Its use in diagnosis and prognostication requires that the test be done by a skilled individual [6] in order to maximise its rather low sensitivity of $67 \%[3,4]$. Accurate interpretation is not solely dependent on ST segment changes during exercise, but other parameters like the presence of chest pain, heart rate at onset of symptoms (if any) and workload done at the end of the test $[4,7,8]$.

\section{Case Presentation}

A 52-year-old male was referred for an ETT from a private health facility. He was known to have hypertension, diabetes, dyslipidaemia and stable angina pectoris for which he was on medication. Though stable on medication, he had however begun experiencing more frequent episodes of chest pain even on minimal exertion. He was therefore referred for the ETT.

ETT was performed using the Bruce protocol. He had a resting pulse rate of $120 \mathrm{bpm}$, with a maximum predicted heart rate (MPHR) of $168 \mathrm{bpm}$. Blood pressure was 150/90 mmHg. Baseline ECG was normal.

After 1 minute and 47 seconds of exercise (stage 1) he clutched his chest, complaining of excruciating pain and demanded that the test be discontinued. A review of the ECG at this time showed horizontal ST segment depressions in leads V4-V6, $2 \mathrm{~mm}$ below the baseline.

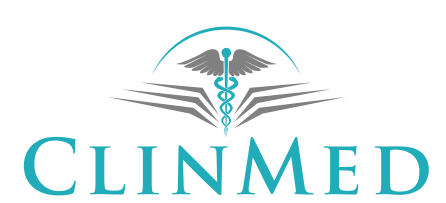

INTERNATIONAL LIBRARY

Citation: Asamoah KT (2021) Maximising the Diagnostic Utility of the Exercise Treadmill Test. Int J Clin Cardiol 8:214. doi.org/10.23937/2378-2951/1410214

Accepted: January 14, 2021: Published: January 16, 2021

Copyright: (C) 2021 Asamoah KT. This is an open-access article distributed under the terms of the Creative Commons Attribution License, which permits unrestricted use, distribution, and reproduction in any medium, provided the original author and source are credited. 
Table 1: Diamond-Forrester classification of chest pain.

\begin{tabular}{|l|l|l|l|l|l|}
\hline Age (years) & Gender & Typical angina & Atypical/probable angina & Non-anginal chest pain & Asymptomatic \\
\hline $\mathbf{3 0 - 3 9}$ & Male & Intermediate & Intermediate & Low & Very low \\
\hline & Female & Intermediate & Very low & Very low & Very low \\
\hline $\mathbf{4 0 - 4 9}$ & Male & High & Intermediate & Intermediate & Low \\
\hline & Female & Intermediate & Low & Very low & Very low \\
\hline $\mathbf{5 0 - 5 9}$ & Male & High & Intermediate & Intermediate & Low \\
\hline & Female & Intermediate & Intermediate & Low & Very low \\
\hline $\mathbf{5} \mathbf{6 0}$ & Both & High & Intermediate & Intermediate & Low \\
\hline
\end{tabular}

Workload done was equal to 4.6 metabolic equivalents of tasks (METs). Blood pressure was $180 / 90 \mathrm{mmHg}$ and his pulse rate was $141 \mathrm{bpm}$, which was $83 \%$ of MPHR. He was immediately given 2 puffs of sublingual nitroglycerin $(0.4 \mathrm{mg})$ and ushered to lie down, as recovery stage monitoring was done. Chest pain intensity gradually reduced and after 4 minutes in the recovery stage, he felt much better and the test was concluded. ST depressions in leads V4 and V5 were however still present, though about $1 \mathrm{~mm}$ deep at this point.

On account of the positive ETT, he was referred for elective coronary angiogram, which was performed four days after the ETT had been done. Coronary angiogram showed multi-vessel disease as follows:

- $75-85 \%$ stenosis of the posterior descending artery

- $75 \%$ stenosis of the right posterolateral artery

- $30 \%$ stenosis of the proximal right coronary artery with some calcification

- Diffuse mild-moderate calcific disease of the left main coronary artery

- $95 \%$ stenosis of the mid-left anterior descending artery with mild calcification

- Varied degrees of diffuse disease in diagonal arteries 1,2 and 3

- $65-70 \%$ stenosis of the mid-portion of the left circumflex artery and the obtuse marginal 2 artery

In view of the angiographic findings, he underwent percutaneous coronary intervention $(\mathrm{PCl})$ with a drug-eluting stent to relieve the stenosis of the left anterior descending artery. His medications were reviewed and he was discharged after a $24 \mathrm{hr}$ observation, with the plan to have another $\mathrm{PCl}$ in a month. He was seen on review 1-week post-PCl and was well. He reported improved exercise tolerance and a complete resolution of the chest pain. He however did not report for the next $\mathrm{PCl}$ session.

\section{Discussion}

Patients for ETT are categorized according to risk/ pretest probability of obstructive CAD based on age, sex and chest pain characteristics using the Diamond-Forrester criteria (Table 1) $[4,9,10]$. Multiple studies have found that this stratification is not often done by reque- sting doctors, resulting in many inappropriate ETTs and consequently, increased expenditure $[4,5,9]$. In order to minimize false negatives/positives, ETTs are recommended for individuals with an intermediate pretest probability $[4,6,9,10]$. It is not recommended that asymptomatic, very low or low risk patients be made to undergo this test [9]. ETT as part of routine medical evaluations is also not recommended as it may give a false sense of safety if negative [11]. Patients with a high probability can be started on treatment without performing this test $[4,9]$. If on medication however and the patient experiences a worsening of his/her symptoms, further investigation is indicated [4].

This patient had been stable for about a year on his anti-anginal medication until shortly before his ETT when his frequency and severity of chest pain increased, as well as his functional limitation. This therefore merited assessment with the ETT.

The exercise done during the test increases myocardial oxygen demand high enough to cause ST segment changes and chest pain induced by myocardial ischaemia if there is a significant coronary obstruction [10]. The minimum heart rate required to draw accurate conclusions is $85 \%$ of the MPHR, which is calculated using the formula "220-age in years" [1]. Higher heart rates afford a higher sensitivity than lower heart rates $[3,4]$. It is therefore recommended that even after patients attain $85 \%$ of MPHR, the test continues for as long possible if there are no features of a positive test, as long as there are no other indications for termination. Evidence of features suggestive of a positive test at heart rates less than $85 \%$ of MPHR, however, suggest significant coronary lesions and portend a poorer prognosis [4]. Submaximal exercise (inability to attain $85 \%$ of MPHR) results in an inconclusive test if there are no changes suggestive of a positive result. The reason for a submaximal test should be sought and if remediable, the test can be repeated after eliminating the cause.

ST segment deviations may occur $[1,4,12]$, and are proportional to the degree of ischaemia induced by the test [4]. ST elevations accurately localize the obstructing lesion to a particular arterial territory, whereas ST depressions are non-localising changes $[1,4,7]$. The association of chest pain with ST segment changes, especially when they occur early within the test (stages 1 and 
2 of the Bruce protocol), is highly suggestive of significant obstructive CAD $[4,7]$. A study showed that $>50 \%$ of such people had triple vessel disease on angiography [4]. Patients with early positive ETTs also tend to have the ST segment changes persisting into the recovery stage, suggesting severe disease $[4,7]$. On occasion, ST segment changes may occur during recovery but not while exercising. There is however no statistically significant difference between the two as long as the ST segment changes meet the criteria for a positive test [12]. Patients who are able to achieve up to 10 METs, i.e. stage 4 of exercise, have a $<10 \%$ likelihood of having multi-vessel disease [4].

The patient being presented experienced an onset of chest pain approximately midway through stage 1 of the test. The ST segment depressions observed on his ECG persisted even after being served with sublingual glyceryl trinitrate and resting for 4 minutes during recovery. In consonance with the literature, the coronary angiogram done showed significant lesions as predicted by the findings during the ETT, most notably the $95 \%$ stenosis of the left anterior descending artery.

The Duke Treadmill Score (DTS) predicts the likelihood of ischaemia due to an obstructive lesion, based on exercise time, ST deviation and chest pain $[4,13,14]$. The duration of exercise at which symptoms and ST segment changes occur, which serves as a surrogate for the workload done i.e. METs, is the most important predictor of cardiovascular mortality [14]. The use of the DTS improves the overall sensitivity of the ETT [4] and is therefore advised to avert performing unnecessary coronary angiograms. The DTS has a significant diagnostic and prognostic value in the assessment of CAD, as well as predicting need for revascularization $[10,13,14]$. Patients with good scores are unlikely to have a significant benefit even if they have revascularization done [4]. This however does not eliminate the need for correlation with clinical history [13]. This patient's DTS of -15 made him a high-risk patient with a 1-year mortality of $8.3 \%$ without any intervention. Coronary angiography showed that this patient had severe CAD, in consonance with the DTS. The DTS must however be used with caution especially in diabetics [15], who due to neuropathy may not perceive pain. This may result in a false classification as low risk using the DTS.

\section{Conclusion}

The ETT is a useful and cost-effective tool for the diagnosis of significant obstructive CAD despite its low sensitivity and specificity, particularly in developing countries that do not have ready access to imaging and other nuclear testing modalities. Workload done during the test is best approximated by the METs achieved and is indicative of the severity of the coronary obstruction. The DTS should be applied to help improve the sensitivity the ETT in diagnosing obstructive CAD. An early positive $E T T$ is highly suggestive of severe CAD with significant coronary artery stenosis and should be followed up with an urgent coronary angiogram and revascularisation. It is also important to remember that people with diabetes may have neuropathy and therefore not experience chest pain as expected.

\section{Financial/Competing Interests}

None to declare.

\section{References}

1. Lim YC, Teo S-G, Poh K-K (2016) ST-segment changes with exercise stress. Singapore Med J 57: 347-353.

2. The top 10 causes of death. 2018. https://www.who.int/ news-room/fact-sheets/detail/the-top-10-causes-of-death (accessed 21/07/2020).

3. Cheezum MK, Subramaniyam PS, Bittencourt MS, Hulten EA, Ghoshhajra BB, et al. (2015) Prognostic value of coronary CTA vs. exercise treadmill testing: Results from the partners registry. Eur Heart J Cardiovasc Imaging 16: 1338-1346.

4. Vaidya GN (2017) Application of exercise ECG stress test in the current high cost modern-era healthcare system. Indian Heart J 69: 551-555.

5. Bilal M, Haseeb A, Arshad MH, Jaliawala AA, Farooqui I, et al. (2018) Frequency and determinants of inappropriate use of treadmill stress test for coronary artery disease. Cureus 10: e2101.

6. Smarż K, Jaxa-Chamiec T, Bednarczyk T, Bednarz B, Eysymontt Z, et al. (2019) Electrocardiographic exercise testing in adults: Performance and interpretation. An expert opinion of the polish cardiac society working group on cardiac rehabilitation and exercise physiology. Kardiol Pol 77: 399-408.

7. Marcadet D-M, Pavy B, Bosser G, Claudot F, Corone S, et al. (2018) French Society of Cardiology guidelines on exercise tests (part 1): Methods and interpretation. Arch Cardiovasc Dis 111: 782-790.

8. Ho PM, Rumsfeld JS, Peterson PN, Masoudi FA, Strunk A, et al. (2007) Chest pain on exercise treadmill test predicts future cardiac hospitalizations. Clin Cardiol 30: 505-510.

9. Silva AML, Armstrong AC, Silveira FJC, Cavalcanti MD, Franca FMF, et al. (2015) Prevalence and factors associated with inappropriate use of treadmill exercise stress test for coronary artery disease: A cross-sectional study. BMC Cardiovascular Disorders 15: 54.

10. Garner KK, Pomeroy W, Arnold JJ (2017) Exercise stress testing: Indications and common questions. Am Fam Physician 96: 293-299.

11. Pais $P(2018)$ Treadmill stress tests should not be part of "routine health check package". Indian Heart J 70: 934-936.

12. Lanza GA, Mustilli M, Sestito A, Infusino F, Sgueglia GA, et al. (2004) Diagnostic and prognostic value of ST segment depression limited to the recovery phase of exercise stress test. Heart 90: 1417-1421.

13. Dzenkeviciute V, Sapoka V, Kasiulevicius V, Rinkuniene E, Steponeniene R, et al. (2017) Value of duke treadmill score in predicting coronary artery lesion and the need for revascularisation. Kardiol Pol 75: 439-444.

14. Salokari E, Laukkanen JA, Lehtimaki T, Kurl S, Kunutsor S, et al. (2019) The Duke treadmill score with bicycle ergometer: Exercise capacity is the most important predictor of cardiovascular mortality. Eur J Prev Cardiol 26: 199-207.

15. Lohiya RV (2014) Duke's treadmill score in diabetics-does it really matter? J Postgrad Med 60: 418. 